\title{
A Morte a Morte De Quncas BerRo d’Água in Italiano: Esiti Positivi e Negativi
}

Mauro Porru*

ABSTRACT: Il presente intervento, partendo dal presupposto che la traduzione è un importante strumento di connessione tra due visioni di mondo, tratta dell'analisi della versione italiana del racconto di Jorge Amado A morte e a morte de Quincas Berro d'Água, tradotta da Elena Grechi, pubblicata da Garzanti nel 1987, a tutt'oggi in circolazione. L'obiettivo principale delle nostre riflessioni è consistito nell'identificare i procedimenti che hanno orientato le scelte traduttive adottate per superare le difficoltà presenti nel testo amadiano, tenendo conto, soprattutto, dell'importanza di un contatto diretto del traduttore con il contesto socio-culturale in cui l'opera originale è nata per poter cogliere con maggior preparazione la struttura del suo linguaggio ed avvicinarsi con più precisione a questa cultura per riuscire a coglierne l'atmosfera.

PAROLE CHIAVE: Jorge Amado; Elena Grechi; traduzione interlinguistica; traduzione source oriented; traduzione target oriented.

RESUMO: Este artigo, partindo do pressuposto de que a tradução é um importante instrumento de conexão entre duas visões de mundo, trata da análise da versão italiana do conto de Jorge Amado A morte e a morte de Quincas Berro d'Água, realizada pela tradutora Elena Grechi e publicada pela editora Garzanti em 1987. O objetivo principal de nossas reflexões consistiu em identificar os procedimentos que orientaram as escolhas tradutórias adotadas para a superação das dificuldades existentes no texto amadiano, levando em conta sobretudo a importância de um contato direto do tradutor com o contexto sociocultural em que a obra original surgiu para que se possa apreender com maior preparo a estrutura de sua linguagem, e aproximar-se com mais precisão dessa cultura para conseguir captar sua atmosfera.

\footnotetext{
* Universidade Federal da Bahia, Salvador, BA (Brasil)-mp43@uol.com.br
} 
PALAVRAS-CHAVE: Jorge Amado; Elena Grechi; tradução interlinguística; tradução source oriented; tradução target oriented.

ABSTRACT: This article, on the assumption that the translation is an important instrument of connection between two world views, analyzes the Italian version of Jorge Amado's tale A morte e a morte de Quincas Berro d'Água, performed by the translator Elena Grechi and published by the Garzanti publisher in 1987. The main objective of our reflections consisted in identifying the procedures that guided the translation choices adopted to overcome the difficulties in Amado's text, considering the importance of a direct contact of the translator with the sociocultural context of the original work to apprehend better the structure of the language, since the proximity with the culture may help capture the ideal atmosphere.

KEYWORDS: Jorge Amado; Elena Grechi; interlinguistic translation; source oriented translation; target oriented translation. 


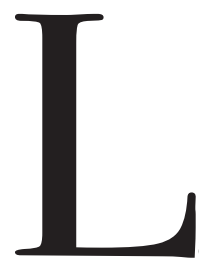

a traduzione è un'attività umana che non si realizza solo con il dominio di tecniche e competenza linguistica. Partendo dalla definizione di traduzione interlinguistica di Roman Jakobson, secondo la quale tradurre consiste nell'interpretazione di segni linguistici per mezzo di un'altra lingua (JAKOBSON, 1995, p. 53), possiamo affermare che è compito del traduttore interpretare il testo originale, con due possibilità: mantenere il testo di arrivo il più vicino possibile a quello di partenza, optando per una traduzione di tipo relativamente tradizionalista, o scegliere una traduzione più vicina alla cultura di arrivo, reinventando espressioni che dialoghino più direttamente con il lettore di arrivo. Rosemary Arrojo (2003) segue la linea interpretativa e va molto oltre, proponendo che il testo originale sia considerato un palinsesto che dovrà essere in un certo qual modo cancellato "in ogni comunità culturale" per essere reinterpretato, dando 
origine ad "un'altra scrittura (o interpretazione, o lettura) dello stesso testo" (ARROJO, 2003, p. 23). Siamo dell'opinione che la cancellazione del testo originale è parziale perché esistono dei limiti per l'interpretazione. Il saggio di Umberto Eco intitolato Riflessioni teorico-pratiche sulla traduzione ci fornisce dei sussidi per le discussioni sull'interpretazione, partendo dai concetti di traduzione source oriented (una traduzione che aspira ad una equivalenza formale con il testo di partenza, cioè la riproduzione naturalmente più vicina della forma, stile, sintassi, morfologia, lessico, eccetera) e del contenuto (significato) del testo di partenza e della traduzione target oriented, che tiene conto soprattutto del pubblico di arrivo, ipotizzando un'equivalenza dinamica, basata sugli effetti equivalenti del testo di arrivo sui lettori/destinatari. Quello che interessa, dunque, è il rapporto tra il messaggio e il lettore/destinatario e, specificamente, la reazione del lettore/destinatario al messaggio, che deve equivalere alla reazione suscitata dal messaggio nel lettore/destinatario originale. Per raggiungere questo scopo bisogna conferire al testo di arrivo una totale naturalità espressiva, prescindendo completamente, in certi casi, dalla forma linguistica usata. Per cui fa parte del processo traduttivo immergersi nell'universo dell'autore e dell'opera che devono essere tradotti, per poter fare delle scelte appropriate e consegnare l'opera a un nuovo pubblico riproducendo, allo stesso tempo, riferimenti ed esperienze appartenenti all'originale. È fondamentale che il traduttore faccia una lettura attenta e approfondita degli elementi culturali presenti nell'opera originale affinché le sue caratteristiche peculiari non si perdano a causa di valutazioni superficiali del traduttore (ECO, 1995, p. 121-146). È di somma importanza rispettare la personalità del testo originale affinché sia mantenuta l'impronta autorale del testo fonte, cioè, nel caso specifico di questo nostro intervento, conservare ciò che fa del testo di Jorge Amado un testo di Jorge Amado.

Basato su questi presupposti teorici analizzeremo la versione italiana del racconto $A$ morte e a morte de Quincas Berro d'Água di Jorge Amado realizzata da Elena Grechi, pubblicata da Garzanti nel 1987, a tutt'oggi in circolazione. La metodologia usata consiste nell'estrarre gli stessi elementi o frammenti di entrambe le opere per esaminare le scelte della traduttrice dinanzi alle difficoltà del testo amadiano. Lo scopo principale è trovare a quali fondamenti teorici la traduzione si è attenuta: se c'è stata una tendenza a conservare le caratteristiche del testo fonte o se, al contrario, c'è stata una dissociazione tra le due opere capace di impoverire il testo di Amado dal punto di vista della ricchezza linguistica e culturale propria di questo autore. Pertanto, la prima fase di questo lavoro, dopo una scrupolosa lettura delle due opere, è consistita nell'effettuare una cernita di espressioni, di lessico e di frasi pertinenti ai nostri obiettivi. Ogni 
elemento selezionato è seguito da un'analisi specifica che offre, in certi casi, suggerimenti di alternative di traduzioni che compensino perdite semantiche o equivoci interpretativi.

Le nostre riflessioni, durante lo svolgersi di questo lavoro, prendono in considerazione soprattutto l'importanza del fatto che il traduttore conosca il contesto socio-culturale da cui emerge l'opera originale; che si informi sul suo linguaggio caratteristico e si avvicini alla concezione di mondo di questa cultura per coglierne l'atmosfera, tenendo presente la grande responsabilità del traduttore con il testo, l'autore del testo tradotto e il pubblico per il quale è stato tradotto, perché è un compito arduo rendere comprensibile e accettabile per i lettori della comunità culturale della lingua di arrivo la lettura del testo di partenza.

Per maggior chiarezza, ci sembra necessaria una breve presentazione del racconto amadiano. L'opera è stata pubblicata per la prima volta nel numero di giugno della rivista Senhor, nel 1959. Secondo Vinicius de Moraes, che ne fece la critica, è "a melhor novela da literatura brasileira", "uma obra-prima". Per il poeta, Jorge Amado è "mais homem que estilo"; feconda la lingua, liberando "as personagens de sua própria teia psicológica" e "as faz saltar, vivas e ardentes, para o lado de cá do livro". Vinicius elogia la crescita di Amado come scrittore, dicendo che si tratta di un

crescimento verdadeiro como a vida, que vem de baixo para cima e sem se recusar às torpitudes; não um crescimento decorativo de araucária, mas de árvore que dá fronde e que dá frutos de polpa, que dá parasitas e dá passarinho: uma gorda e resinosa mangueira. E que melhor comparação, para o deleite da leitura desse baiano da peste, que o comer mangas, os dentes mordendo fundo a carne da fruta, a terebintina escorrendo pelo queixo no seu amarelo pungente, a gulodice de enxugar o caroço até o fim... [...] Saí da leitura dessa extraordinária novela [...] com uma sensação de bem-estar físico e espiritual como só dão os prazeres do copo e da mesa, quando se está com sede ou fome, e os da cama quando se ama. (MORAES, 2001, p. 12-13).

Infatti, il testo amadiano possiede un linguaggio molto semplice che evita le esagerazioni dei puristi della Lingua e riproduce la naturalezza dei discorsi quotidiani di differenti classi sociali. Amado, osservando attentamente quello che accade per le strade di Bahia, inserisce un vocabolario più regionalizzato, includendo gerghi ed espressioni della cultura locale. L'autore 
si colloca nella posizione di un osservatore che scrive in terza persona, riproducendo i fatti e le loro interpretazioni senza interferire. I vicoli, le strade, gli spazi abitati dalla gente povera, umile, felice ed esclusa della vecchia Salvador vengono praticamente dissezionati per essere analizzati e messi in discussione dai lettori che devono possedere una certa malizia, ironia e umore per aderire all'autore che affronta il tema della morte in modo divertente, doloroso e ironico.

La situazione iniziale della narrativa è la vera morte di Quincas nella topaia in cui ha abitato durante la sua vita dissoluta. Partendo da questo fatto, in retrospettiva, si svolge il passato del protagonista in due tappe distinte: presso la famiglia, come persona "normale", e insieme agli emarginati come un vagabondo volontario e dichiarato. Poi, in prospettiva, si accompagna il funerale e la passeggiata del morto, fino a quando cade in mare.

I personaggi principali sono: Joaquim Soares da Cunha o Quincas Berro d'Água (il protagonista); la moglie Otacília; la figlia Vanda; il genero Leonardo Barreto; i fratelli Eduardo e Marocas; gli amici Curió, Negro Pastinha, Cabo Martim e Pé-de-vento; e la sua amante Quitéria do Olho Arregalado.

Il racconto, diviso in 12 capitoli, narra le varie morti di Quincas. Prima di diventare Quincas, Joaquim Soares da Cunha era un impiegato statale, padre e marito esemplare fino al giorno in cui va in pensione. Da quel momento in poi si sbarazza della famiglia, della rispettabilità, dei conoscenti, degli amici e della tradizione. Diventa un vagabondo alcolizzato e giocatore d'azzardo. Lascia la famiglia per vivere con prostitute, ubriaconi, marinai, giocatori, ladruncoli e delinquenti dei quartieri malfamati di Salvador. Sazia la sua sete con la cachaça e si riposa sulle spalle accoglienti delle prostitute. Rispettato e ammirato dai suoi nuovi compagni di avventura è il paisinho, saggio e consigliere, sempre pronto per un'altra baldoria o sbornia. La sua scelta per la sfrenatezza rappresenta il grido terribile di un uomo dominato e accerchiato da pregiudizi di ogni tipo, che un giorno rompe tutti i legami e invoca urlando la propria libertà. Muore solitario su un letto immondo e la cui morte scatena tutto un processo di riconoscimento/disconoscimento da parte della famiglia reale e da quella adottata. Gli amici durante la veglia si ubriacano e, sbronzi, decidono di far fare al "defunto" un ultimo giro per il quartiere malfamato in cui abitano. La passeggiata include bordelli e bettole e finisce su un saveiro dove ci sono donne e cibi. All'improvviso arriva una tempesta e il corpo di Quincas cade in mare. Così, egli, rinunciando alla famiglia, cambiando ambiente e abitudini, muore una prima volta; nella solitudine della sua stanzetta lurida, avvolto in degli stracci, smaltendo la sua ultima sbornia, muore una seconda volta; cadendo in mare senza lasciare nessuna traccia fisica della sua esistenza, muore una terza volta. 
Cominciamo ora a esaminare la versione italiana del racconto. Il primo elemento da analizzare è il titolo: A morte e a morte de Quincas Berro d'Água. Elena Grechi lo traduce Le due morti di Quincas Acquaiolo. Traducendo A morte e a morte in Le due morti (As duas mortes) la traduttrice compie una semplificazione che sminuisce molto l'impatto del sintagma principale che vuole sottolineare le varie versioni della morte di Quincas e non comunicare semplicemente che è morto due volte, come suggerisce la semplificazione Le due morti. La scelta di tradurre Berro d'Água in Acquaiolo ci sembra totalmente equivocata perché tutta la forza semantica dell'originale si perde completamente. In italiano il termine "Acquaiolo" significa "quello che vende l'acqua da bere". Lui in effetti grida "acqua", ma lo fa per vendere questo prodotto, per invitare le persone a comprarlo, a usarlo. Un grido, quindi, diametralmente diverso dal grido disumano di Quincas che scopre con orrore di aver bevuto acqua pensando che fosse cachaça. Sicuramente, sarebbe stato meglio lasciare il titolo in originale e fornire la spiegazione nel capitolo VII in cui si chiarisce l'origine di questo epiteto. Così la frase "Desde aquele dia em que passou a ser chamado Berro d'Água" (AMADO, 1995, p. 45), potrebbe essere tradotta in italiano: "Dal giorno in cui gli era stato affibbiato l'epiteto Berro (grido) d'Água (dell'acqua)", o mettendo in nota o in un glossario la spiegazione. Tradurre "Berro d'Água" in "Acquaiolo", oltre a perdere completamente la forza semantica dell'originale, può far credere al lettore italiano che Quincas sia un venditore d'acqua fino a quando, nel racconto, non si spiega l'origine di questo epiteto.

Per quanto riguarda l'epigrafe, "Cada um cuide de seu enterro, impossível não há (Frase derradeira de Quincas Berro d'Água, segundo Quitéria que estava ao seu lado)" (AMADO, 1995, p. IX), è stata tradotta: "Ognuno si occupi del proprio funerale, cose impossibili non ce n'è (Ultima frase di Quincas Acquaiolo, a detta di Quitéria che si trovava al suo fianco)" (AMADO, 1987, p. 8).

La prima parte dell'epigrafe è stata tradotta letteralmente: "cada qual" "ognuno", "cuide" "si occupi" (dal verbo riflessivo occuparsi, che è l'esatto corrispondente italiano del verbo “cuidar”) “do seu enterro", “del proprio funerale”. La scelta dell'aggettivo possessivo di terza persona proprio invece di suo, è corretto dal punto di vista grammaticale. Infatti, in italiano si deve preferire l'aggettivo possessivo "proprio" all'aggettivo possessivo "suo" quando il soggetto è un pronome indefinito come nel caso di "ognuno", ma questa scelta grammaticalmente corretta introduce una rigidità formale assente nell'originale, rivelando fin dall'inizio la mancanza di una linea definita di scelte traduttive. Difatti, se a volte si eseguono rigidamente 
le regole grammaticali, come in questo caso, anche se impoveriscono il testo, o forniscono una connotazione sbagliata alle parole e ai sintagmi, o irrigidiscono l'emozione, l'umore e la poesia del testo originale. In altri casi, invece, come nella seconda parte dell'epigrafe, la traduttrice usa costruzioni testuali tipiche toscane, traducendo "impossível não há" in "cose impossibili non ce n'è", mentre la traduzione grammaticamente corretta sarebbe "cose impossibili non ce ne sono", concordando il soggetto plurale "cose" con il verbo "esserci" nella terza persona plurale, usando la particella pronominale "ne" nella sua accezione neutra. Una scelta traduttiva più pertinente, capace di conservare in lingua italiana il tono leggero e ironico del testo di partenza potrebbe essere: "Ognuno si occupi del suo funerale. Nulla è impossibile" (nada è impossível).

Quanto alla nota dell'epigrafe “(Frase derradeira de Quincas Berro d'Água, segundo Quitéria que estava ao seu lado)" (AMADO, 1995, p. IX), Grechi, per tradurre in italiano la preposizione "segundo", anziché usare l'esatto corrispondente "secondo", preferisce impiegare la locuzione poco usata e eminentemente formale "a detta di" (dal neutro plurale latino dicta - le cose dette). La locuzione prepositiva "ao seu lado" è stata tradotta "al suo fianco", modificando semanticamente il senso dell' espressione brasiliana. "Al suo fianco" in italiano significa "stare vicino a qualcuno" nel senso di appoggiarlo, di sostenerlo, e corrisponde alla locuzione portoghese "estar do seu lado", mentre l'esatto corrispondente dell'espressione brasiliana "estar ao seu lado", in italiano, è la locuzione prepositiva "accanto a". Una migliore scelta traduttiva, pertanto, sarebbe: "Ultima frase di Quincas Berro d'Água, secondo Quitéria che gli stava accanto" (que estava junto dele).

Per quanto riguarda i nomi propri di persona, la traduttrice ha preferito conservare quelli originali, ad eccezione di Marocas che diventa Marietta (Mariazinha). Gli epiteti, ad eccezione di Curió, sono stati tradotti in italiano, creando uma serie di equivoci e perdite semantiche. Per esempio, Negro Pastinha è stato tradotto Negro Riccioletto, perdendo, così, totalmente la forza semantica dell'originale. Il termine "pastinha", in portoghese, indica un tipo di pettinatura in cui i capelli, unti con una specie di brillantina gelatinosa, creano un riporto ondulato sulla fronte. "Ricciolino", in italiano, è il diminutivo di "ricciolo" (cacho), una parola che non definisce nessun tipo di pettinatura e tanto meno una acconciatura che ha bisogno di un tipo di brillantina gelatinosa per farla. La parola "ricciolino" è usata normalmente nell'accezione di testa piena di riccioli e indica soprattutto un bambino dai capelli ricci.

I termini tipici della cultura baiana, della culinaria e delle feste religiose rimangono in originale, ma le note esplicative dimostrano, quasi tutte, la poca dimestichezza della traduttrice con questi aspetti della cultura baiana. 
Esaminiamo ora la traduzione di parole ed espressioni tipiche baiane, appartenenti al gergo della malavita e altre create dalla fantasia di Amado.

Nel primo capitolo, in cui il narratore anticipa al lettore i misteri e le lacune sulla morte di Quincas, riguardo alla notizia della sua morte si legge: "largamente falada nas ladeiras e becos escusos" (AMADO, 1995, p. 2). Nella traduzione italiana, questa frase diventa "ampiamente commentata per vicoli e stradette" (AMADO, 1987, p. 9). Così le ladeiras perdono la loro forza semantica diventando "ruelas" e i becos escusos divengono "ruas estreitas", perdendo quel senso di mistero e di illecito insito nell'originale. Una migliore scelta traduttiva potrebbe essere: "largamente ripetuta nelle calate e nei vicoli tortuosi". L'espressione "largamente ripetuta" attenuerebbe il tono formale contenuto in "ampiamente commentata"; la parola "calata", che in italiano definisce un luogo attraverso il quale si scende, conserverebbe in parte la forza semantica del termine "ladeira"; la parola "vicoli" è l'esatto corrispondente italiano di "becos" e l'aggettivo "tortuoso", che in italiano significa pieno di curve, sinuoso e ambiguo, manterrebbe, almeno in parte, il senso recondito, misterioso e illecito dell'aggettivo "escuso".

Sempre nel primo capitolo, quando si descrive Joaquim Soares da Cunha come un serio impiegato, si legge: "bebedor de cachaça tão longa e conversada" (AMADO, 1995, p. 4). Tradotta in italiano diventa "bevitore di cachaça dalla sbornia così tarda e conversatoria" (AMADO, 1987, p. 10). Questa scelta ci sembra particolarmente infelice. Allontanandosi totalmente dal significato che l'espressione ha in portoghese, Grechi traduce tão longa "dalla sbornia cosi tarda", usando il sostantivo femminile popolare "sbornia" al lato dell'aggettivo "tarda", usato esclusivamente nel linguaggio letterario, nel senso di "pigro, non sollecito" e per estensione "lento, che arriva in ritardo divenendo inutile, e conversada con il neologismo "conversatoria" (dal verbo "conversare"), creando una espressione "sbornia conversatoria", che in italiano non ha alcun senso, oltre ad essere spaventosamente cacofonica. Una scelta migliore potrebbe essere "una persona capace di bere molta cachaça (o acquavite) chiacchierando senza sbronzarsi".

Nel secondo capitolo, quando si parla dei commenti sui momenti finali della vita di Quincas, si legge: "Até um folheto com versos de pé quebrado foi composto pelo repentista Cuica de Santo Amaro e vendido largamente" (AMADO, 1995, p. 5). Nella versione italiana diventa: "Fu perfino composta dal cantastorie Cuíca di Santo Amaro e largamente venduta, una ballata sull'argomento, in settenari zoppi" (AMADO, 1987, p. 11). Ritradotta in portoghese questa frase diventa: "Foi até composta pelo cantor popular Cuíca de Santo Amaro e largamente vendida, uma balada sobre este argumento, em setenários mancos". Così, il folheto diventa una 
"balada", os versos de pé quebrado, diventano "setenários mancos" e il repentista un "cantor de histórias populares". Dunque, la parola folheto ha un suo corrispondente in italiano, "foglietto", che significa "piccolo foglio, foglio stampato per annunciare notizie e un piccolo giornale". Quanto a versos de pé quebrado, in italiano, esiste l'espressione "rima zoppa" che indica una rima difettosa che non segue una metrica, incompleta o priva di alcuno dei suoi elementi, che giustificherebbe l'espressione "versi zoppi”. Il repentista, in italiano, è "il poeta estemporaneo, il poeta che improvvisa versi", un termine troppo lungo che interromperebbe il ritmo della frase. Per mantenere il significato e il ritmo della frase originale, sarebbe stato meglio tradurre: "Lo stesso Cuíca di Santo Amaro, che tanto bene improvvisa versi zoppi, ha stampato un foglietto su questa storia e ne ha venduti tantissimi".

Sempre nel secondo capitolo, Joaquim, prima di diventare Quincas, era un impiegato esemplare "jamais visto num botequim, de cachaça caseira e comedida" (AMADO, 1995, p. 6). Questa frase viene tradotta in italiano: "mai visto in una bettola, bevitore morigerato e casalingo" (AMADO, 1987, p. 11). La traduttrice, di nuovo, per tradurre letteralmente la frase, usa termini come "bettola", "morigerato" e "casalingo" che ingessano il testo originale. Reinterpretando il testo di partenza, si riuscirebbe a mantenere il suo ritmo e il suo significato intrinseco, traducendo: "mai visto in un bar, perché beveva poco e in casa".

Nel terzo capitolo, quando si parla del cadavere di Quincas, si legge: "Cadáver para necrotério, para ir no rabecão da polícia, servir depois aos alunos da Faculdade de Medicina nas aulas práticas, ser finalmente enterrado em cova rasa, sem cruz nem inscrição" (AMADO, 1995, p. 14). Questo brano è stato tradotto: "Un cadavere da obitorio, buono per esser portato via dalla camionetta della polizia, per poi servire agli studenti di medicina in aula di anatomia ed essere infine sepolto sotto due palmi di terra, senza croce né lapide" (AMADO, 1987, p. 15). Come si può constatare nella traduzione, sia il ritmo che la forza semantica del testo sono abbastanza pregiudicati. Si nota nella traduttrice una grande tendenza a specificare e fissare termini che danneggiano la riproduzione nella lingua di arrivo delle particolarità dell'originale. "Rabecão", termine brasiliano di grande effetto che indica il veicolo in cui si trasportano i cadaveri, diventa nella traduzione "una camionetta", un tipo di veicolo della polizia per trasportare dei prigionieri, ma mai dei cadaveri, perdendo, così, tutta la forza semantica del termine originale. Sarebbe più appropriato usare il termine italiano "furgone", che indica un veicolo chiuso della polizia e anche la vettura usata dalle pompe funebri per trasportare il feretro. Il sintagma "os alunos da Faculdade de Medicina" è semplificato in "studenti di medicina". Il termine generico "aulas 
práticas", diventa un termine specifico "aula di anatomia" (inoltre il termine italiano "aula" non corrisponde a quello brasiliano poiché in italiano "aula" indica lo spazio fisico dove avvengono le lezioni, "a sala", e non la propria lezione, "a aula") perdendo parzialmente la forza semantica dell'originale. Corretta è la scelta dell'espressione "sotto due palmi di terra" per tradurre "em cova rasa", visto che conserva tutta la forza semantica dell'originale. La sostituzione del termine "inscrição" in "lapide" dimostra di nuovo la preoccupazione della traduttrice di specificare e fissare i termini della lingua di partenza anche quando esistono gli esatti corrispondenti nella lingua d'arrivo.

Un'altra possibile traduzione più vicina al testo originale potrebbe essere: "Un cadavere da obitorio, da furgone della polizia, da finire in mano agli studenti della Facoltà di Medicina per le esercitazioni pratiche, da essere sepolto, alla fine, sotto due palmi di terra, senza croce né iscrizione", nella quale l'uso ripetuto della preposizione semplice "da", nella sua accezione di complemento di finalità, intento e uso, definisce correttamente quale potrebbe essere la fine del cadavere di Quincas.

Nel quinto capitolo, quando si descrive una lunga discussione su come comunicare al resto della famiglia e ai conoscenti la morte di Quincas, troviamo la frase: "Tia Marocas arrotou, o bucho farto e o coração também" (AMADO, 1995, p. 25). Questa stessa proposizione è stata tradotta: "Zia Marietta sospirò. Lo stomaco pieno le allargava il cuore" (AMADO, 1987, p. 20). La trasformazione di un arroto (rutto) in "sospiro", nella prima parte della frase, rivela una vera e propria censura del testo, visto che in italiano esiste l'esatto corrispondente "ruttare". Il termine "bucho", che in Brasile significa popolarmente "barriga, ventre", è stato tradotto educatamente "stomaco", anche se in italiano esiste il termine popolare "pancia" che tradurrebbe con più esattezza l'originale. Far sì che lo stomaco pieno aumenti il cuore di zia Marocas non ha alcun senso e modifica completamente il testo originale in cui si dice semplicemente che zia Marocas ha la pancia piena e anche il cuore. Una traduzione appropriata sarebbe: "Zia Marocas fece un rutto, la pancia gonfia, il cuore pure", in cui l'aggettivo "gonfio", che in senso figurato significa "pieno", "turgido", può essere usato sia con la parola "pancia" che con la parola "cuore", riproducendo tutta la forza semantica dell'originale.

Nel sesto capitolo si legge:

Dez anos levara Joaquim essa vida absurda. "Rei dos vagabundos da Bahia", escreviam sobre ele nas colunas policiais das gazetas, tipo de rua citado em 
crônicas de literatos ávidos de fácil pitoresco, dez anos envergonhando a família, salpicando-a com a lama daquela inconfessável celebridade. O "cachaceiro mor", "o filosofo esfarrapado da rampa do Mercado", "O senador das gafieiras". (AMADO, 1995, p. 31-32).

Questo brano è stato tradotto:

Per dieci anni Joaquim aveva condotto quella vita assurda, "Re dei vagabondi di Bahia", era spesso citato nella cronaca nera, dato in pasto a letterati avidi di pittoresco facile, che parlavano di lui nelle loro cronache come dell'archetipo del vagabondo di strada, dieci anni a coprir di vergogna la famiglia, sporcandola col fango di quella discutibile celebrità. Il "grappaiolo maggiore di Bahia", "il filosofo straccione della rampa del Mercato, "il senatore delle balere". (AMADO, 1987, p. 23).

La prima frase è stata tradotta correttamente, dato che l'espressione "condurre una vita" è l'esatto corrispondente dell'espressione brasiliana "levar uma vida". Il resto del testo è stato cambiato ancora una volta senza motivo. La frase "escreviam sobre ele nas colunas policiais das gazetas" viene semplificata in "era spesso citato nella cronaca nera" (corretta l'espressione "cronaca nera", esatto corrispondente in italiano di "crônicas policiais"). La proposizione "tipo de rua citado em crônicas de literatos ávidos de fácil pitoresco", viene, al contrario, accresciuta di elementi che, ancora una volta, tendono a definire esageratamente i fatti, divenendo: "dato in pasto a letterati avidi di pittoresco facile, che parlavano di lui nelle loro cronache come dell'archetipo del vagabondo di strada". La parte del brano "dez anos envergonhando a família, salpicando-a com a lama daquela inconfessável celebridade" viene tradotta in modo enfatico: "dieci anni a coprir di vergogna la famiglia, sporcandola col fango di quella discutibile celebrità". Una scelta particolarmente infelice è aver tradotto "O cachaceiro mor de Salvador", con "Il grappaiolo maggiore di Bahia", ricorrendo alla creazione del neologismo "grappaiolo" (da grappa), particolarmente cacofonico, che, inoltre, può trarre in inganno il lettore, visto che può essere anche attribuito a chi produce la grappa. "O filosofo esfarrapado da rampa do mercado" è stato tradotto letteralmente con l'esatto corrispondente italiano "il filosofo straccione della rampa del Mercato". "O senador das gafieiras" è stato tradotto con molta pertinenza "il senatore delle 
balere", poiché la parola "balera" definisce, in italiano, un locale da ballo semplice, popolare, dove si danzano i ritmi tipici del liscio.

Una traduzione più attinente all'originale potrebbe essere: "Per dieci anni Joaquim aveva condotto quella vita assurda. 'Re dei vagabondi di Bahia'. Si scriveva di lui nella cronaca nera dei giornali; personaggio folclorico citato nelle cronache di letterati, avidi di facili note pittoresche, dieci anni di vergogna per la famiglia, infangata da quella inconfessabile celebrità, 'Il più grande bevitore di cachaça di Salvador', 'il filosofo straccione della rampa del Marcato', 'il senatore delle balere"'.

Nell'ultimo paragrafo del settimo capitolo, quando si racconta una delle tante avventure di Quincas, si legge: "Não se atirara ele, ainda há poucos dias, velho e bêbado, como um campeão sem medo, em defesa de Clara Boa, quando dois jovens transviados, filhos da puta, das melhores famílias, quiseram surrá-la numa farra no castelo de Viviana?" (AMADO, 1995, p. 43). Il brano è stato tradotto: "Non si era forse gettato ancora giorni prima, lui vecchio e sbronzo, come un campione senza paura, contro due giovinastri figli di buona donna di ottime famiglie, in difesa di Clara-la-Bona, che loro volevano picchiare durante un'orgia in casa di Viviana?" (AMADO, 1987, p. 31). La traduzione ancora una volta censura il testo originale, alleggerendo alcune espressioni e modificandone altre senza motivo. Si percepisce di nuovo la tendenza della traduttrice a particolarizzare e censurare, compromettendo la possibile riproduzione in lingua italiana della naturalezza del testo amadiano. Infatti, l'inclusione, senza necessità, dei pronomi personali di terza persona "lui e loro" e lo spostamento dell'inciso "em defesa de Clara Boa" rendono il testo più pesante e meno spontaneo. Molto adeguata la scelta dell'aggettivo "sbronzo" per tradurre "bêbado": si tratta di un aggettivo usato nel linguaggio popolare romano, ma perfettamente comprensibile in tutta Italia che definisce una persona ubriaca. Adeguata è anche la scelta di tradurre "Clara Boa" con il suo esatto corrispondente Clara-la-Bona, che in dialetto romano, comprensibile in tutta Italia, ha la stessa accezione brasiliana di "mulher boa, gostosa". Appropriata è anche la scelta del termine "giovinastri” per tradurre jovens transviados. Questo termine, composto dal sostantivo "giovane" e il suffisso peggiorativo "astro", definisce esattamente un jovem transviado, senza principi morali. "Filhos da puta" diventa "figli di buona donna", una forma più delicata dell'esatto corrispondente italiano "figli di puttana". L'espressione "das melhores famílias", viene tradotta "di ottime famiglie", che è il corrispondente in italiano, salvo che l'espressione più usata è al singolare "di ottima famiglia". A "farra no castelo de Viviana" cambia in "un'orgia in casa di Viviana". 
In questo caso, la traduzione si allontana abbastanza dall'originale, dato che semanticamente si sovraccarica il termine farra (festa animata), che diventa "un'orgia" e si svuota totalmente il termine castelo, usato in Brasile per definire un postribolo, un bordello e che diventa "casa". Così un'altra possibile traduzione più attinente all'originale potrebbe essere: "Non si era forse gettato ancora pochi giorni prima, vecchio e sbronzo, come un campione senza paura, in difesa di Clara-la-Bona, contro due giovinastri figli di puttana di ottima famiglia, che volevano picchiarla durante una festa nel bordello di Viviana?".

Nel nono capitolo, quando si descrive l'attrazione di Negro Pastinha per il corpo ben fornito di Tia Marocas, si legge: "Não gostava dessas magricelas, cuja cintura a gente não pode apertar. Se Negro Pastinha encontrasse essa madama na praia, fariam misérias a dois, bastava olhar para ela e logo se via sua qualidade" (AMADO, 1995, p. 66). Il brano è stato tradotto così: "Non gli garbavano quelle seccanicce che non gli si può neppure strizzare la vita. Se Negro Ricciolino avesse incontrato la madama sulla spiaggia, avrebbero fatto follie, loro due; bastava guardarla per capire che era una donna di qualità" (AMADO, 1987, p. 40). Sicuramente, la traduzione di questo brano è la più discutibile, perché riesce ad eliminare tutta la splendente vivacità del linguaggio amadiano e la sua intensità erotica. La prima scelta inadeguata è l'uso del verbo "garbare" per tradurre il verbo "gostar". Si tratta, in effetti, quasi di un toscanismo (la traduttrice è toscana), dato che "garbare" è un verbo che non si usa nel linguaggio popolare e che solo in Toscana è utilizzato come sinonimo del verbo "piacere" (verbo semanticamente corrispondente al verbo "gostar"). "Magricelas" è stato tradotto "seccanicce", con un termine sconosciuto alla maggior parte degli italiani, di probabile origine toscana, comprensibile perché possiede la radice dell'aggettivo "secco", che nel linguaggio popolare oltre che seco può significare anche magro, oppure si può trattare di un neologismo creato dalla traduttrice. Sarebbe stato molto più appropriato usare l'esatto corrispondente italiano "magroline". La frase "cuja cintura a gente nem pode apertar" diventa "che non gli si può neppure strizzare la vita". Il verbo "strizzare" significa "spremere qualcosa finché tutto il liquido ne esca", o nel caso di "strizzare gli occhi", significa "ammiccare", "stringere le palpebre per vedere meglio o per difendersi dalla luce eccessiva". Per cui, anche in questo caso si tratta di un toscanismo; infatti, nel dialetto toscano il verbo "strizzare" è sinonimo di "stringere" (apertar). Per tradurre l'espressione "fariam misérias" la traduttrice usa il modo di dire italiano "fare follie", che riproduce parzialmente la forza semantica dell'originale, perché non ha la stessa intensità erotica. Sarebbe più adeguato usare l'espressione "farne di cotte e di crude" che si avvicina abbastanza al tono erotico 
dell'originale. La frase "bastava olhar para ela e logo se via sua qualidade" in italiano diventa "bastava guardarla per capire che era una donna di qualità". La versione italiana, trasformando le qualità fisiche attraenti di zia Marocas in qualità morali, perde totalmente la sua forza semantica e la sua ironia sensuale. Sarebbe stato meglio usare la costruzione "bastava guardarla per vedere le sue qualità", che possiede, in italiano, la stessa ironia sensuale del testo di partenza.

Una traduzione più appropriata, quindi, sarebbe "Non gli piacevano quelle magroline, che non si potevano neanche stringere alla vita. Se Negro Pastinha avesse incontrato quella madama sulla spiaggia, insieme ne avrebbero fatte di cotte e di crude, bastava guardarla per vedere le sue qualità".

Nell'undicesimo capitolo, Quitéria sussurra parole d'amore a Quincas:

Por que pregar susto na gente, Berrito desgraçado? Tu bem sabe que tenho o coração fraco, o médico recomendou que eu não me aborrecesse. Cada idéia tu tem, como posso viver sem tu, homem com parte com o tinhoso? Tou acostumada com tu, as coisas malucas que tu diz, tua velhice sabida, teu jeito sem jeito, teu gosto de bondade. Por que tu me fez isso hoje? - e tomava a cabeça ferida na peleja, beijava-lhe os olhos de malícia. (AMADO, 1995, p. 91).

Questo brano è stato tradotto così in italiano:

Perché spaventarci così, Acquaiolino disgraziato? Lo sai che ho il cuore debole, il medico ha raccomandato di non darmi dispiaceri. Ti vengono certe idee, a tu, come faccio a vivere senza di tu, uomo che ha fatto un patto con il demonio? Sono abituata con tu, con le cose pazze che tu dice, alla tua vecchiaia sapiente, ai tuoi modi senza modo, al tuo gusto di bontà. Perché oggi m'hai fatto questo?" e prendeva tra le mani il volto ferito nella lotta, baciava gli occhi maliziosi. (AMADO, 1987, p. 53-54).

Il tentativo di riprodurre nella lingua di arrivo gli errori di concordanza verbale e di uso dei pronomi personali di seconda persona dell'originale ci sembra particolarmente infelice. Anche il meno colto degli italiani non userebbe le costruzioni create dalla traduttrice. La traduzione della frase "o médico recomendou que eu não me aborrecesse" in "il medico ha raccomandato di non darmi dispiaceri” sostituisce, senza nessuna necessità, il pronome personale soggetto di 
prima persona $\mathrm{eu}$ (io) con il pronome personale di prima persona con valore di complemento di termine "mi”, spostando la responsabilità dell'azione da Quitéria a Quincas. La traduzione della frase "tua velhice sabida" in "tua vecchiaia sapiente" caratterizza un classico caso di "falso amico"; infatti, in questo contesto, l'aggettivo sabido in portoghese corrisponde all'aggettivo italiano "furbo", "astuto", "scaltro", e non "sapiente". La frase "teu gosto de bondade" è stata tradotta letteralmente "al tuo gusto di bontà", con una costruzione che in italiano suona un poco strana. Forse sarebbe stato meglio interpretare questa frase, per renderla più comprensibile al lettore italiano, esternando il suo significato intrinseco, come, per esempio, "quella tua voglia di essere buono". Vengono fatti inoltre dei cambiamenti superflui, come "cabeça ferida" (testa ferita) che diventa "il volto ferito"; "peleja" in "lotta" nonostante in italiano esistano delle parole come "zuffa", "mischia", "rissa" che esprimono lo stesso concetto di "peleja". Particolarmente infelice, nella sua estrema cacofonia, la traduzione di "Berrito" in "Acquaiolino".

Una traduzione più attinente capace di non svuotare completamente il vigore poetico del testo fonte potrebbe essere: "Perché ci hai fatto spaventare così, Berrito disgraziato? Lo sai che ho il cuore debole, il medico mi ha raccomandato di evitare dispiaceri. Ma come ti vengono certe idee? Come faccio a vivere senza di te, uomo che hai fatto il patto col diavolo? Sono abituata con te, con le cose pazze che dici, con la tua scaltra vecchiaia, i tuoi modi senza modo, la tua voglia di essere buono. Perché oggi m'hai fatto questo? - e prendeva tra le mani la testa ferita nella rissa e gli baciava gli occhi maliziosi”.

Per quanto riguarda i versi che concludono il racconto, alla fine del dodicesimo capitolo,

No meio da confusão

Ouviu-se Quincas dizer:

"- Me enterro como entender

Na hora que resolver.

Podem guardar seu caixão

Pra melhor ocasião.

Não vou deixar me prender

Em cova rasa no chão"

E foi impossível saber

$\mathrm{O}$ resto da oração.

(AMADO, 1995, p. 95-96). 
la traduttrice, cercando di conservare la metrica, usa delle parole poco ricorrenti come "bailamme" o inesatte come "cassone" al posto di "bara", che offuscano la vivacità del testo originale:

In mezzo a quel bailamme

S'udì Quincas dichiarare:

"Mi seppellisco come voglio

E al momento che mi pare.

Mettete via la bara

Per un'altra occasione

Non mi lascerò chiudere

Sotto terra in un cassone".

E fu impossibile udire

Il resto dell'orazione.

(AMADO, 1987, p. 55).

Anche in questo caso sarebbe stato meglio interpretare il testo originale per cercare, nel limite del possibile, di riprodurre nella lingua di arrivo la vivacità del testo fonte, come per esempio: "In quella confusione si udì Quincas gridare:/ "Mi faccio seppellire come e quando mi pare./ Mettete pure via la bara che sta là/ per una ben migliore opportunità./ Perché sotto terra non ci voglio andare/ e questa, cari miei, è la pura verità"./ Da questo punto in poi mai saper si potrà/ Come Quincas concluse la sua estrema volontà".

Da questa nostra analisi risulta che la traduttrice, durante il processo traduttivo, ha usato diversi strumenti che variano dalla traduzione letterale alla creazione di termini di vario tipo.

Per quanto riguarda le espressioni e altri elementi linguistici, la traduttrice, in generale, ha avuto esiti parzialmente positivi quando ha scelto il sistema target oriented che tende ad avvicinare il testo originale alla comunità culturale di arrivo, ma le sue soluzioni source oriented, nella maggior parte dei casi, hanno falsato il contesto originale, pregiudicandone l'interpretazione. Si è constatato, inoltre, durante questa analisi, che la traduttrice, in certi casi, rimane entro i limiti in cui il traduttore può muoversi con una certa libertà e giocare con il testo originale senza però svincolarsi completamente da esso. In molti altri casi, invece, si perde in interpretazioni inopportune, come, per esempio, modificare, nella versione italiana, elementi 
linguistici della cultura brasiliana, soprattutto quando si tratta di espressioni volgari usate dai personaggi. Grechi le censura tutte. Si notano, poi, la mancanza di letture complementari e uno studio approfondito degli elementi culturali dell'opera fonte, assolutamente necessari per creare i significati del processo interpretativo in cui consiste la traduzione.

Per concludere possiamo affermare che la traduttrice, possedendo una scarsa conoscenza degli elementi culturali dell'opera fonte, e non riuscendo a "sentire" e a "impadronirsi" del linguaggio dei vari personaggi, ingessa e ingrigisce il vivace e colorato linguaggio del racconto di Amado con una traduzione italiana troppo garbata, prevedibile e a volte di difficile lettura.

\section{Riferimenti bibliografici}

AMADO, J. A morte e a morte de Quincas Berro d'Água. 69. ed. Rio de Janeiro: Record, 1995. . La doppia morte di Quincas l'acquaiolo. Trad. Elena Grechi. Milano: Garzanti, 1987.

ARROJO, R. O signo desconstruído. Implicações para a tradução, a leitura e o ensino. 2. ed. Campinas: Pontes, 2003.

ECO, U. Riflessioni teorico-pratiche sulla traduzione. In NERGAARD, S. (org.). Teorie contemporanee della traduzione. Milano: Bompiani, 1995, p. 121-146.

JAKOBSON, R. Aspetti linguistici della traduzione. In NERGAARD, S. (org.). Teorie contemporanee della traduzione. Milano: Bompiani, 1995, p. 51-62.

MORAES, V. A morte e a morte de Quincas Berro d'Água. In AMADO, J. A morte e a morte de Quincas Berro d’Água. 77. ed. Rio de Janeiro: Record, 2001, p. 12-13.

Recebido em 30/03/2016

Aprovado em 15/05/2016 